\title{
Exact results for supersymmetric quantum mechanics on the lattice
}

\author{
David Baumgartner* and Urs Wenger \\ Albert Einstein Center for Fundamental Physics \\ Institute for Theoretical Physics \\ University of Bern \\ Sidlerstrasse 5 \\ CH-3012 Bern \\ Switzerland \\ E-mail: baumgartaitp.unibe.ch, wengeraitp.unibe.ch
}

We discuss $\mathscr{N}=2$ supersymmetric quantum mechanics on the lattice using the fermion loop formulation. In this approach the system naturally decomposes into a bosonic and fermionic sector. This allows us to deal with the sign problem arising in the context of broken supersymmetry due to the vanishing of the Witten index. Employing transfer matrix techniques we obtain exact results at finite lattice spacing and are hence able to study how the continuum limit is approached. In particular, we determine how supersymmetry is restored and how, in the case of broken supersymmetry, the goldstino mode emerges.

The XXIX International Symposium on Lattice Field Theory

July 11-16, 2011

Squaw Valley, California, USA

* Speaker. 


\section{Introduction}

Calculating nonperturbative properties of supersymmetric theories on the lattice encounters various difficulties related to the fact that the discretisation of space-time explicitly breaks supersymmetry and violates Leibniz' rule. Moreover, the vanishing of the Witten index in the context of spontaneous supersymmetry breaking leads to a fermion sign problem which makes straightforward numerical simulations impossible. While the restoration of supersymmetry can sometimes be achieved in the continuum limit of the lattice theory, e.g. by fine tuning or by constructing $Q$-exact discretisations [1], a solution to the sign problem is not easy to find. A possible way out has been proposed in [2, 3, 4]. It is based on the fermion loop formulation which can be simulated without critical slowing down even when a massless goldstino mode is present.

In this work, we apply the fermion loop formulation to $\mathscr{N}=2$ supersymmetric quantum mechanics for superpotentials yielding broken or unbroken supersymmetry. Using transfer matrix techniques we are able to obtain exact results for partition functions and various observables at finite lattice spacing. We investigate how the supersymmetric spectrum is recovered in the continuum limit and how the goldstino mode emerges in the case of broken supersymmetry. In these proceedings we confine ourselves to the presentation of results obtained using a Wilson type discretisation together with the appropriate fine tuning of counterterms, although results using a $Q$-exact discretisation have been derived as well.

\section{Supersymmetric quantum mechanics on the lattice}

The continuum action of $\mathscr{N}=2$ supersymmetric quantum mechanics can be written as

$$
S=\int d t\left[\frac{1}{2}\left(\frac{d \phi(t)}{d t}\right)^{2}+\frac{1}{2} P^{\prime}(\phi(t))^{2}+\bar{\psi}(t)\left(\frac{d}{d t}+P^{\prime \prime}(\phi(t))\right) \psi(t)\right]
$$

with one real bosonic coordinate $\phi$, two anticommuting fermionic coordinates $\bar{\psi}$ and $\psi$, and a generic superpotential $P(\phi)$. The derivative of the superpotential $P^{\prime}(\phi)$ is taken with respect to $\phi$, $P^{\prime}(\phi) \doteq \frac{\partial P(\phi)}{\partial \phi}$. For periodic boundary conditions $(P B C)$ the action is invariant under two supersymmetry transformations $\delta_{1,2}$ :

$$
\begin{aligned}
\delta_{1} \phi & =\psi \bar{\varepsilon}, & \delta_{2} \phi & =\bar{\psi} \varepsilon, \\
\delta_{1} \psi & =0, & \delta_{2} \psi & =\left(\frac{d \phi}{d t}-P^{\prime}\right) \varepsilon, \\
\delta_{1} \bar{\psi} & =\left(\frac{d \phi}{d t}+P^{\prime}\right) \bar{\varepsilon}, & \delta_{2} \bar{\psi} & =0,
\end{aligned}
$$

with two Grassmann valued parameters $\varepsilon$ and $\bar{\varepsilon}$. Note that for supersymmetric quantum mechanics it is the form of the superpotential $P(\phi)$ which determines the supersymmetry breaking pattern. If the highest power of $P(\phi)$ is even (odd), supersymmetry is unbroken (broken). A main feature of supersymmetry is the degeneracy between the energy levels in the bosonic and the fermionic sector. For unbroken supersymmetry, however, there is one single unpaired energy level at zero energy, i.e., a unique ground state, either in the bosonic or in the fermionic sector. This is in contrast to the case of broken supersymmetry, where the lowest energy levels in both sectors are degenerate and lifted above zero. In addition, there is a zero energy goldstino mode which mediates between the two degenerate ground states. 
The supersymmetry breaking pattern can also be partly infered from the Witten index. It is formally defined as

$$
W \equiv \lim _{\beta \rightarrow \infty} \operatorname{Tr}\left[(-1)^{F} \exp (-\beta H)\right],
$$

where $F$ denotes the fermion number operator and $H$ is the Hamiltonian of the system. Essentially, $W$ counts the difference between the number of bosonic and fermionic zero energy states and its vanishing provides a neccessary but not sufficient condition for supersymmetry breaking. $W$ can also be written more explicitely as

$$
W=\lim _{\beta \rightarrow \infty}\left[\operatorname{Tr}_{b} \exp (-\beta H)-\operatorname{Tr}_{f} \exp (-\beta H)\right]=\lim _{\beta \rightarrow \infty}\left[Z_{0}-Z_{1}\right]=\lim _{\beta \rightarrow \infty} Z_{P B C},
$$

where $\operatorname{Tr}_{b, f}$ denote the traces over the bosonic and fermionic states. $Z_{0,1}$ are the partition functions in the $F=0,1$ sectors and $Z_{P B C}$ is the one with periodic boundary conditions. In the language of field theory, the latter can be calculated via

$$
Z_{P B C}=\int \mathscr{D} \phi \mathscr{D} \bar{\psi} \mathscr{D} \psi \exp (-S)=\int_{-\infty}^{\infty} \mathscr{D} \phi \operatorname{det} D(\phi) \exp \left(-S_{\phi}\right) .
$$

In the last step, the fermions have been integrated out yielding the fermion matrix determinant $\operatorname{det} D$ and the bosonic part of the action $S_{\phi}$. In this representation, the origin of a fermion sign problem becomes evident when supersymmetry is broken: a vanishing Witten index requires the determinant $\operatorname{det} D$ to be indefinite.

\subsection{Lattice formulation}

For the construction of a lattice version of the model, we follow Golterman and Petcher [5] and employ the same lattice derivative for the bosons as for the fermions. To avoid fermion doublers, we use the Wilson lattice derivative with Wilson parameter $r=1$. In one dimension this simplifies to the backward derivative $\left(\Delta^{-} f\right)_{x}=f_{x}-f_{x-1}$ and the discretised action explicitly reads

$$
S_{L}=\sum_{x}\left[\frac{1}{2}\left(P^{\prime}\left(\phi_{x}\right)^{2}+2 \phi_{x}^{2}\right)-\phi_{x} \phi_{x-1}+\left(1+P^{\prime \prime}\left(\phi_{x}\right)\right) \bar{\psi}_{x} \psi_{x}-\bar{\psi}_{x} \psi_{x-1}\right]
$$

Due to radiative corrections the lattice theory is, however, not guaranteed to yield a supersymmetric theory in the continuum limit. The corrections can be accounted for either by adding a suitable counterterm $\frac{1}{2} \sum P^{\prime \prime}$ to the action $[5,6]$, which restores the supersymmetries in the continuum limit, or by adding the surface term $\sum P^{\prime}\left(\Delta^{-} \phi\right)[7,8,9]$ resulting in a $Q$-exact action. The latter construction preserves a particular combination of the supersymmetries $\delta_{1,2}$ exactly even at finite lattice spacing and hence guarantees the correct continuum limit without any fine tuning.

To circumvent the sign problem discussed above, we make use of the fermion loop formulation $[2,3,4]$. The basic idea here is to exactly rewrite the exponential of the fermion degrees of freedom as a power series to all orders. Upon integration of the fermion fields, the nilpotency of the Grassman variables yields a constraint on the oriented fermionic bond occupation numbers $n_{x}^{f}=0,1$ between the sites $x$ and $x-1$ related to the fermion hopping term $\bar{\psi}_{x} \psi_{x-1}$, and on the monomer occupation numbers $m_{x}^{f}=0,1$ stemming from the term $\left(1+P^{\prime \prime}\left(\phi_{x}\right)\right) \bar{\psi}_{x} \psi_{x}$. The constraint is given by

$$
m_{x}^{f}+\frac{1}{2}\left(n_{x}^{f}+n_{x+1}^{f}\right)=1 \quad \forall x
$$


and allows only two fermion configurations: $\left\{m_{x}^{f}=1, n_{x}^{f}=0, \forall x\right\}$ with fermion number $F=0$, and $\left\{m_{x}^{f}=0, n_{x}^{f}=1, \forall x\right\}$ with fermion number $F=1$. For PBC the latter receives an additional minus sign relative to the former due to the fermion loop. As a consequence, the partition function naturally decomposes into a bosonic and fermionic contribution $Z_{0}$ and $Z_{1}$, in accordance with eq.(2.2). It is this decomposition which eventually allows to take care of the fermion sign problem.

In addition to the fermion bonds and monomers we also introduce non-oriented bonds for the bosonic degrees of freedom, with the corresponding bosonic bond occupation numbers $n_{x}^{b} \in \mathbb{N}^{0}$ [4]. Depending on the symmetries of the action, these bosonic bond configurations may obey certain constraints. By summing over these constrained configurations $\left\{n_{x}^{b}\right\}$ we obtain the locally factorised partition functions with fixed fermion number $F=0,1$,

$$
Z_{F}=\sum_{\left\{n_{x}^{b}\right\}} \prod_{x} \frac{1}{n_{x}^{b} !} Q_{F}\left(N_{x}\right)
$$

where the local weights $Q_{F}$ are defined as

$$
Q_{F}(N)=\int d \phi \phi^{N} \mathrm{e}^{-\frac{1}{2}\left(P^{\prime}(\phi)^{2}+2 \phi^{2}\right)}\left(1+P^{\prime \prime}(\phi)\right)^{1-F}
$$

with the bosonic site occupation number $N_{x}=n_{x}^{b}+n_{x+1}^{b}$. The $Q$-exact discretisation requires additional types of bosonic bonds, but still leads to a locally factorised partition function.

\subsection{Transfer matrix}

The dimensionality of the system allows a further reformulation in terms of a transfer matrix between states defined on the dual lattice. Each state is characterised by the fermion bond occupation number number $n^{f}$ and the boson bond occupation number $n^{b}$, i.e. $\left|n^{f}, n^{b}\right\rangle$. Since the fermion number is conserved the transfer matrix has a block structure consisting of the two matrices $T_{m^{b}, n^{b}}^{F=0,1}$ which take the system from state $\left|F, n^{b}\right\rangle$ to $\left|F, m^{b}\right\rangle$. To be specific, the transfer matrix elements are given by

$$
T_{m^{b}, n^{b}}^{F}=\frac{1}{\sqrt{m^{b} !}} \frac{1}{\sqrt{n^{b} !}} Q_{F}\left(m^{b}+n^{b}\right) .
$$

In order to keep the size of the matrices finite, we introduce a cutoff on the maximal bosonic bond occupation number. Keeping it of the order $\mathscr{O}\left(10^{2}\right)$ turns out to be sufficient to render all results independent of the cutoff.

In terms of these transfer matrices, the partition function for a system with $L_{t}$ lattice sites is calculated in each sector $F$ according to

$$
Z_{F}=\operatorname{Tr}\left[\left(T^{F}\right)^{L_{t}}\right]
$$

These partition functions can then be combined to $Z_{P B C}=Z_{0}-Z_{1}$ and $Z_{a P B C}=Z_{0}+Z_{1}$ for $P B C$ and antiperiodic boundary conditions $(a P B C)$, respectively. The construction via the transfer matrices allows the straightforward calculation of various observables, such as correlation functions, Ward identities and mass gaps. The latter are directly associated with the eigenvalues of the transfer matrices. If we denote the eigenvalues of $T^{F}$ by $\lambda_{0}^{F}>\lambda_{1}^{F}>\ldots$, the $k$-th bosonic mass gap in the sector $F$ can be calculated as

$$
m_{b}^{F, k}=-L_{t} \cdot \log \left(\lambda_{k}^{F} / \lambda_{0}^{F}\right), \quad k=1,2, \ldots
$$



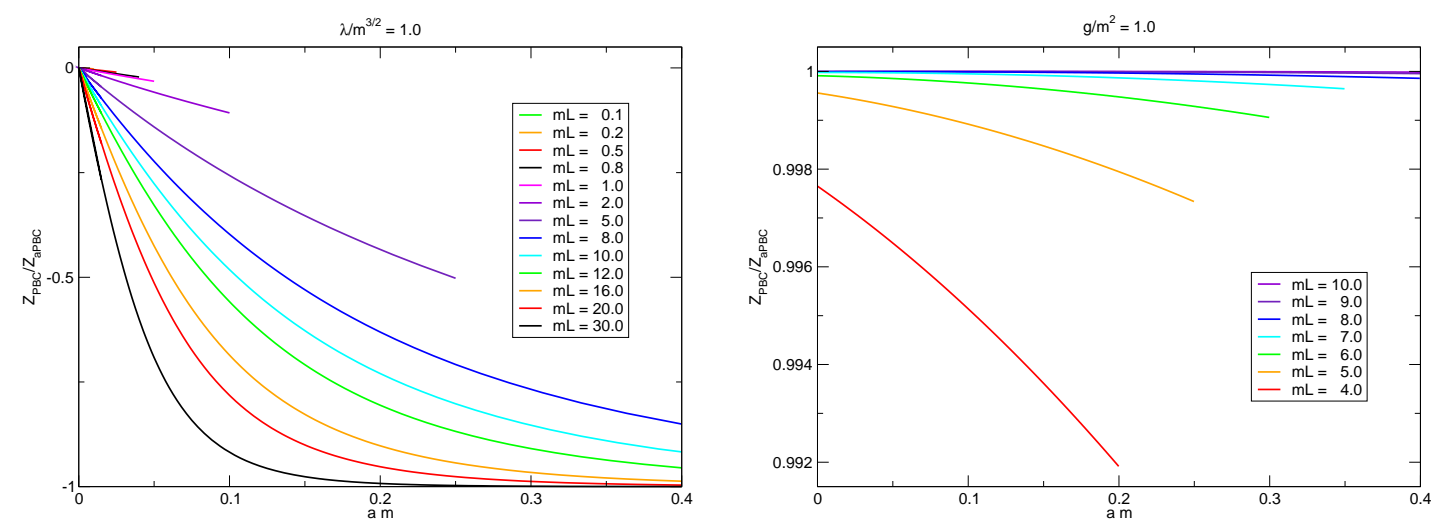

Figure 1: The Witten index $W=Z_{P B C} / Z_{a P B C}$ versus the lattice spacing for broken (left plot) and unbroken supersymmetry (right plot) at various values of the inverse temperature $m L$. The continuum limit corresponds to $a m \rightarrow 0$.

whereas the $k$-th fermionic energy gap is given by

$$
m_{f}^{F, k}=-L_{t} \cdot \log \left(\lambda_{k}^{1-F} / \lambda_{0}^{F}\right), \quad k=0,1, \ldots
$$

The transfer matrix approach can, of course, be generalised straightforwardly to any kind of discretisation of the action eq.(2.1), in particular also to the $Q$-exact discretisation.

\section{Results}

We now present the results for the action (2.3) with the counterterm using the techniques introduced above. For our calculations, we use the superpotential $P_{u}(\phi)=\frac{1}{2} m \phi^{2}+\frac{1}{4} g \phi^{4}$ as an example with unbroken supersymmetry and $P_{b}(\phi)=-\frac{m^{2}}{4 \lambda} \phi+\frac{1}{3} \lambda \phi^{3}$ as an example for which the supersymmetry is broken. The calculations are performed at coupling strengths $g / \mathrm{m}^{2}=1.0$ and $\lambda / m^{3 / 2}=1.0$, respectively, thus we are clearly in a regime where perturbation theory is not applicable. For a system with aPBC for the fermion the temporal extent of the lattice is inversely related to the temperature $T$ of the system, such that $m L \rightarrow \infty$ corresponds to the zero temperature limit. Finally, the continuum limit is reached by taking $L_{t} \rightarrow \infty$.

\subsection{Witten index}

The Witten index is determined by the quantity $Z_{P B C} / Z_{a P B C}=\left(Z_{0}-Z_{1}\right) /\left(Z_{0}+Z_{1}\right)$. It measures the relative weight between the bosonic and fermionic sectors $Z_{0}$ and $Z_{1}$, respectively. In the system with broken supersymmetry both ground states are equally favourable, yielding $Z_{P B C} / Z_{a P B C}=0$ in the zero temperature limit. Of course, the degeneracy between the two ground states is broken at finite lattice spacing, so one expects a Witten index $W= \pm 1$ in the limit $T \rightarrow 0$ at fixed $a$. It turns out that for our choice of parameters, the fermionic ground state has a slightly lower energy at finite $a$ leading to $W=-1$ in the $T \rightarrow 0$ limit, $c f$. left plot in figure 1 . This is true for any finite

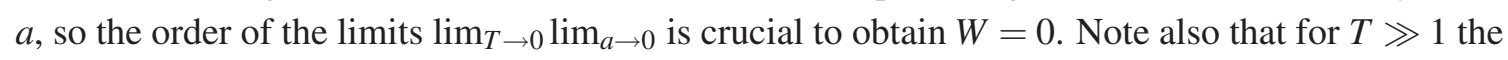



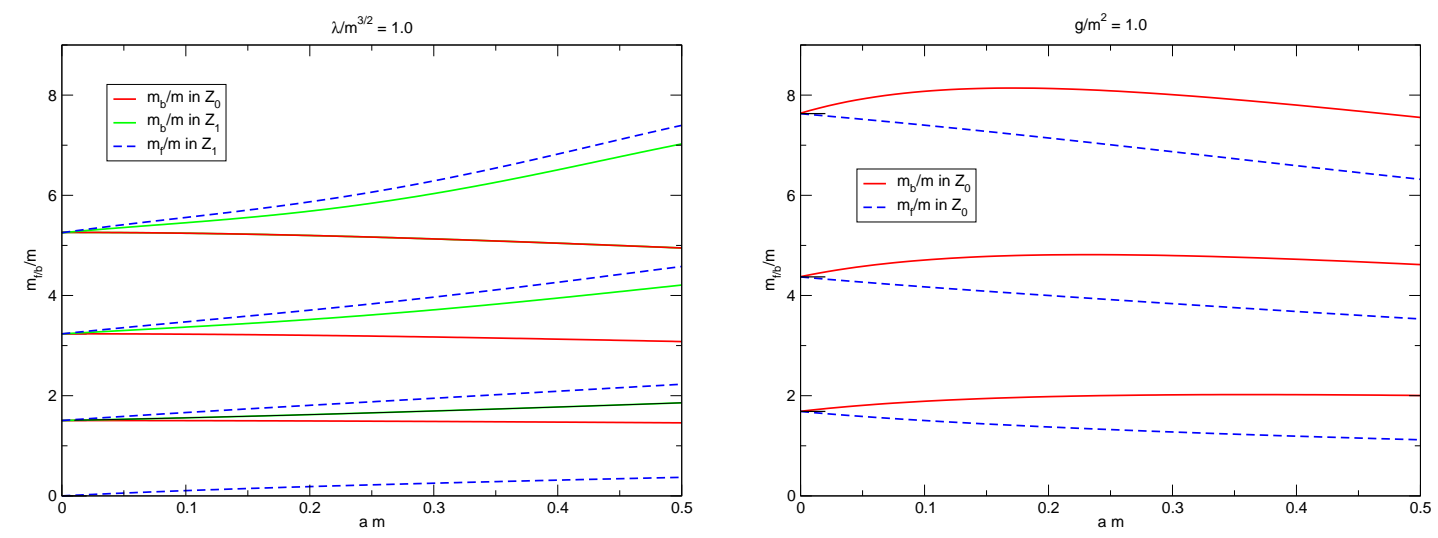

Figure 2: Bosonic and fermionic mass gaps $m_{b}$ and $m_{f}$ versus the lattice spacing $a$, all expressed in units of the bare mass $m$. Results for broken and unbroken supersymmetry are displayed in the left and right panel, respectively. Note that for broken supersymmetry the zero energy Goldstino mode emerges in the continuum limit.

Witten index tends to zero at any finite $a$, in accordance with the counting of the states in eq.(2.2) at finite temperature.

In the situation with unbroken supersymmetry the system is forced to occupy the single unique ground state in the zero temperature limit, yielding $Z_{P B C} / Z_{A P B C}=+1$ or -1 . Note that for our specific choice of parameters the ground state is bosonic, hence $W=+1$. However, while one finds that the index is pushed away from 0 for $T \gg 1$ as before, in the limit $T \rightarrow 0$ it will always go to 1 at any finite $a, c f$. right plot in figure 1 . So it turns out that for unbroken symmetry, the order of the two limits $\lim _{T \rightarrow 0}$ and $\lim _{a \rightarrow 0}$ is not relevant.

\subsection{Mass gaps}

It is also interesting to study how the energy or mass gaps approach the continuum limit. In figure 2 we show the results for the lowest few masses as a function of the lattice spacing $a$, everything expressed in units of the bare mass $m$, for broken (left plot) and for unbroken supersymmetry (right plot). Since we extract the mass gaps from the eigenvalues of the transfer matrices, the results are obtained directly in the limit $T \rightarrow 0$. As a consequence, for broken supersymmetry, where there are two degenerate ground states in the continuum, it makes sense to calculate bosonic excitations $m_{b}$ both in the $F=0$ and $F=1$ sector. The plots illustrate nicely how the supersymmetry in the spectrum, i.e. the degeneracies between the bosonic and fermionic excitations, are restored in the continuum limit. Furthermore, when the supersymmetry is broken one expects a zero energy fermionic excitation, the goldstino mode, which is responsible for the fact that $Z_{P B C}=0$. From the plot it becomes clear how the lattice acts as a regulator for the goldstino mode and, as a consequence, also for the vanishing Witten index $W$, hence allowing to give meaning to (finite) observables even in the system with PBC. Finally, we make the observation that the leading lattice artefacts of the spectral mass gaps are all $\mathscr{O}(a)$ except for $m_{b}$ in the $F=0$ sector when the supersymmetry is broken. In that case they are $\mathscr{O}\left(a^{2}\right)$. 


\section{Conclusions}

We have presented exact results for $\mathscr{N}=2$ supersymmetric quantum mechanics on the lattice using the fermion loop formulation and corresponding transfer matrices. With these techniques we are able to study in detail how the supersymmetric spectrum is recovered in the continuum limit and how the Witten index is regularised on the lattice.

In the loop formulation the partition function naturally separates into bosonic and fermionic contributions and this is crucial for containing the fermion sign problem in supersymmetric systems with broken supersymmetry. The transitions between the bosonic and fermionic sectors are controlled by the (would-be) goldstino mode which becomes massless only in the continuum limit. Since massless fermion modes can be efficiently simulated with the fermion loop algorithm proposed in [2,3] our approach provides a way to circumvent the sign problem. Indeed, results from Monte Carlo simulations of $\mathscr{N}=2$ supersymmetric quantum mechanics have already been presented in [4] and in this work we have provided the corresponding exact results using transfer matrices.

It is also interesting to apply our approach to higher dimensions where it allows to investigate the spontaneous breaking of supersymmetry nonperturbatively and from first principles. In particular, the approach can be applied to supersymmetric Wess-Zumino models [4] in $d=2$ dimensions and first results from simulations of the $\mathscr{N}=1$ model including one Majorana fermion and one scalar field have been presented at this conference [10].

\section{References}

[1] S. Catterall, Twisted lattice supersymmetry and applications to AdS/CFT, PoS LATTICE2010 (2010) 002, [arXiv: 1010.6224$]$.

[2] U. Wenger, Efficient simulation of relativistic fermions via vertex models, Phys. Rev. D80 (2009) 071503, [arXiv:0812.3565].

[3] U. Wenger, Simulating Wilson fermions without critical slowing down, PoS LAT2009 (2009) 022, [arXiv:0911.4099].

[4] D. Baumgartner and U. Wenger, Simulation of supersymmetric models on the lattice without a sign problem, PoS LATTICE2010 (2010) 245, [arXiv: 1104 . 0213].

[5] M. F. L. Golterman and D. N. Petcher, A local interactive lattice model with supersymmetry, Nucl. Phys. B319 (1989) 307-341.

[6] J. Giedt, R. Koniuk, E. Poppitz, and T. Yavin, Less naive about supersymmetric lattice quantum mechanics, JHEP 12 (2004) 033, [hep-lat/ 0410041 ].

[7] S. Catterall and E. Gregory, A lattice path integral for supersymmetric quantum mechanics, Phys. Lett. B487 (2000) 349-356, [hep-lat/ 0006013$].$

[8] S. Catterall, Lattice supersymmetry and topological field theory, JHEP 0305 (2003) 038, [hep-lat/0301028].

[9] G. Bergner, T. Kaestner, S. Uhlmann, and A. Wipf, Low-dimensional supersymmetric lattice models, Annals Phys. 323 (2008) 946-988, [arXiv : 0705.2212 ].

[10] D. Baumgartner, K. Steinhauer, and U. Wenger, Supersymmetry breaking on the lattice: the $N=1$ Wess-Zumino model, PoS LATTICE2011 (2011) 253, [arXiv:1111.6042]. 